\title{
Prácticas pedagógicas para el desarrollo de competencias ciudadanas
}

\section{Teaching Practices in Developing Citizenship Skills}

\author{
Elizabeth Leonor Zambrano (*) elizabethzambrano24@gmail.com \\ (*) Universidad del Magdalena \\ (Recibido: 23 de mayo de 2016; Aceptado para su publicación: 3 de noviembre de 2016)
}

Cómo citar: Zambrano, E. L. (2018). Prácticas pedagógicas para el desarrollo de competencias ciudadanas. Revista Electrónica de Investigación Educativa, 20(1), 69-82 https://doi.org/10.24320/redie.2018.20.1.1409

\section{Resumen}

En este artículo se presentan los resultados obtenidos del análisis de la aplicación de la prueba en competencias ciudadanas en un grupo de estudiantes de la Universidad del Magdalena (Colombia) después de haber recibido las prácticas pedagógicas constructivas (estudio de casos) y lúdicas (juegos) para la adquisición de competencias ciudadanas. El estudio es cuantitativo, de diseño cuasi-experimental, con aplicación de pre-prueba y post-prueba, con un grupo de control que recibió la intervención de prácticas pedagógicas expositivas (clases magistrales) y un grupo experimental que recibió la intervención de prácticas pedagógicas lúdicas y prácticas constructivas. Las pruebas paramétricas realizadas a los datos obtenidos permiten concluir que existen diferencias significativas a favor de los alumnos que emplearon las prácticas pedagógicas lúdicas y constructivas para el desarrollo de competencias ciudadanas. Esto sugiere la necesidad de seguir aplicando prácticas pedagógicas eficaces para el desarrollo de estas competencias en los estudiantes universitarios.

Palabras clave: Competencias ciudadanas, prácticas pedagógicas, educación superior, juegos didácticos, estudio de caso.

\section{Abstract}

This paper presents the results of an analysis of the application of a test of citizenship skills on a group of students from the University of Magdalena (Universidad de Magdalena) after they received teaching practices that were both constructive (case studies) and playful (games) to acquire citizenship skills. The study is quantitative with a quasi-experimental, pretest-posttest design, a control group that received explanations (lectures) and an experimental group that received playful and constructive teaching practices. The parametric tests performed on the data obtained lead to the conclusion that there are significant differences in favor of students who used playful and constructive teaching practices to develop citizenship skills. This points to the need to continue applying effective teaching practices to develop these skills in university students. 


\section{Introducción}

Los álgidos problemas que vive Colombia han ocasionado el deterioro de la conducta, llevando a los individuos a una escasa o nula participación en los escenarios que le competen, al empleo de estereotipos arraigados en sus culturas que dificultan el reconocimiento del otro, y a la poca tolerancia en las relaciones interpersonales, etc. Frente a estas problemáticas, la educación ha planteado estándares en competencias ciudadanas, los cuales sólo cubren los programas de Educación Básica y Secundaria, por lo que se hace necesario establecerlos en el nivel universitario, a fin de dar respuesta a los fines y objetivos de una Educación Superior pertinente. Para su aplicación en el aula deben estar acordes a prácticas pedagógicas activas, con un grado de profundidad y complejidad mayor en cada uno de los ámbitos, y con conocimientos que permitan la integración de las destrezas de pensamiento y competencias propias. Reafirmando lo anterior, Giroux (1992) considera que a través del aula se puede cambiar la formación pasiva que se le ha dado al ciudadano mediante la modificación de las prácticas pedagógicas empleadas por el profesor, utilizando metodologías de enseñanza que cuestionen el conocimiento y le permitan al estudiante participar responsablemente en su aprendizaje.

En la presente investigación se considera a las prácticas pedagógicas como aquellas estrategias, instrumentos y acciones que el docente realiza en el aula para guiar el proceso de enseñanza aprendizaje, con la pretensión de desarrollar en el educando diversas competencias, específicamente las competencias ciudadanas (cognitivas, comunicativas, emocionales e integradoras). Estas prácticas han de ser observables por otros y permitir a los docentes realizar meta-cognición sobre su enseñanza a fin de replantear su quehacer educativo y lograr el interés y la motivación por parte de los estudiantes (Zambrano, Rivera, Fernández y González, 2014). Además, estas prácticas deben estar orientadas por el currículo y la experiencia del educador; en el primer caso con el fin de articular los diferentes componentes de los procesos formativos y de interacción que se desarrollan en el aula (Briones, 2004), y en el segundo, dando coherencia a los conocimientos y competencias que docentes desean que el estudiante desarrolle, contribuyendo en la renovación de las metodologías de los docentes (Rangel, 2015).

Existen prácticas pedagógicas que se utilizan en el proceso de formación de los estudiantes universitarios, entre ellas: prácticas expositivas, constructivas, de profundización y lúdicas. En esta investigación se aplicaron prácticas lúdicas (juegos) y constructivas (estudio de casos) a un grupo de tratamiento, mientras que a otro grupo sólo se le aplicaron prácticas expositivas (clases magistrales) para demostrar cómo las primeras prácticas desarrollaban las competencias ciudadanas entre los alumnos.

Las competencias ciudadanas son un conjunto de conocimientos, actitudes y habilidades -cognitivas, emocionales y comunicativas- que articuladas entre sí hacen que el ciudadano democrático esté dispuesto a actuar de manera constructiva y justa en la sociedad (Ministerio Nacional de Educación [MEN], 2004, p. 8). Esto conlleva un desarrollo como ser en el instante en que interactúa con otros y con la naturaleza, requiriendo del individuo conocimientos, habilidades y valores para lograr su compromiso y responsabilidad ante dilemas de la sociedad. Por ello, el desarrollo de las competencias ciudadanas se presenta como alternativa pedagógica a aplicar frente a las exigencias de los cambios coyunturales que requiere Colombia.

Carrillo (2013) define las competencias ciudadanas como la capacidad para participar en múltiples contextos, ámbitos sociales y laborales en los que se desempeña el individuo; coincidiendo con Villarini (2010), quien indica que las competencias ciudadanas son el desarrollo de conceptos, actitudes y destrezas que se requieren para ser un miembro activo, reflexivo, crítico y responsable de un pueblo que busca organizarse como comunidad política democrática.

Los elementos que componen las competencias ciudadanas son las acciones y las habilidades. Las acciones son consideradas como la capacidad y la actitud de actuar acorde con los principios democráticos (Instituto Colombiano para el Fomento de la Educación Superior [ICFES], 2005). Por otro lado, las habilidades son entendidas como "herramientas" causales del desempeño de una persona dentro de un contexto en el que se encuentra inmerso; éstas forman parte de las competencias y están 
relacionadas con el aprendizaje y la educación de los individuos en todas las etapas de la vida (Climent, 2010). Las habilidades que conforman las competencias ciudadanas son las integradoras, comunicativas, emocionales y cognitivas. Las habilidades integradoras se definen como la capacidad de articular todas las habilidades a través de acciones y actitudes ciudadanas (ICFES, 2004; 2005).

Existen evidencias de que las acciones ciudadanas estarían incluidas en las relaciones interpersonales, ya que permiten comprender a los otros, tener autoconocimiento, potencializando actuar en la vida de un modo más eficaz (Gardner, 1983). Por otro lado, Cabrera (2002, como se cita en Tey, Vilá y Martín, 2014) expresa que las competencias ciudadanas, dentro de las cuales están las acciones, cobran importancia cuando se concibe como una práctica.

De acuerdo a los hallazgos anteriores se propone la hipótesis 1: Las acciones ciudadanas son significativamente mayores en el grupo experimental que ha recibido prácticas pedagógicas constructivas y lúdicas, que en el grupo de control que ha utilizado prácticas pedagógicas expositivas.

Las habilidades comunicativas son la actitud y capacidad para establecer una comunicación asertiva, para lo cual el sujeto conoce los derechos fundamentales del individuo como pautas para generar participación y la comunicación constructiva y efectiva (Soriano, 2006), entendido esto como aquellos contextos para el ejercicio de la participación democrática (MEN, 2006). Por lo tanto, los ambientes democráticos son fundamentales en la formación de sujetos capaces de realizar acciones autónomas, armónicas y responsables, basadas en la interiorización de valores como la convivencia, la tolerancia, la participación y la paz (Mieles y Alvarado, 2012); el rol del maestro es crear estos ambientes de aprendizaje, en los cuales haya comunicación constante (Cano, 2004).

De acuerdo a los hallazgos mencionados se propone la hipótesis 2: Los ambientes democráticos son significativamente mayores en el grupo experimental que ha recibido prácticas pedagógicas constructivas y lúdicas, que en el grupo de control que ha desarrollado prácticas pedagógicas expositivas.

Las emociones, como elemento dentro de las competencias ciudadanas, son las habilidades útiles para identificar, controlar, comprender y expresar adecuadamente nuestros sentimientos y emociones, y conocer el porqué de los mismos (Ugarriza, 2001). Por otro lado, la empatía, entendida como la capacidad de percatarse, comprender, apreciar y experimentar sentimientos iguales o parecidos a los que está sintiendo otra persona (Ugarriza y Pajares, 2005). Las emociones y la empatía pueden desarrollarse a través de la educación, ya que capacitan al individuo para que adopte comportamientos que tengan presentes los principios de prevención y desarrollo humano (Bisquerra, 2000).

Con base en lo anterior, se propone:

Hipótesis 3: Las emociones son significativamente mayores en el grupo experimental que ha recibido prácticas pedagógicas constructivas y lúdicas, que en el grupo de control que ha realizado prácticas pedagógicas expositivas.

Hipótesis 4: La empatía es significativamente mayor en el grupo experimental que ha recibido prácticas pedagógicas constructivas y lúdicas, que en el grupo de control que ha realizado prácticas pedagógicas expositivas.

Por último, las habilidades cognitivas se entienden como la capacidad para plantear hipotesis y reflexionar para el ejercicio de la ciudadanía, fortaleciendo la capacidad del pensamiento crítico y la habilidad para la resolución de problemas (Gross y Contreras, 2006). Otros autores las definen como la capacidad de cuestionar y evaluar la validez de cualquier creencia, información y decisiones propias y de los demás (Ruiz y Chaux, 2005), considerándolo como el producto del proceso evolutivo y de las experiencias de los individuos (Baumeister y Heatherton, 1996). Para su aplicación se requieren tres elementos: el desarrollo de destrezas de razonamientos, de las actitudes y de la autorregulación (Ruiz y Chaux, 2005). Tomando como referencia a los autores mencionados, se propone la siguiente hipótesis 5: El pensamiento crítico es significativamente mayor en el grupo experimental que ha recibido prácticas 
pedagógicas constructivas y lúdicas, que en el grupo de control que ha implementado prácticas pedagógicas expositivas.

\section{Método}

Para testar las hipótesis planteadas se llevó a cabo un diseño cuasi experimental, con pre-prueba y postprueba, para dos cursos de educación general universitaria. Al grupo de control se le aplicó la práctica pedagógica expositiva (clases magistrales) y al grupo experimental se le aplicaron las prácticas pedagógicas lúdicas (siete juegos) y constructivas (siete estudios de casos), después de haberle aplicado la pre-prueba.

Para la aplicación de las prácticas pedagógicas se realizaron las siguientes actividades: revisión documental relacionada con las competencias ciudadanas y la construcción de las prácticas lúdicas y constructivas (su estructura y componentes) y los contenidos de la cátedra (los derechos y deberes ciudadanos, el Estado, la ciudadanía, la resistencia civil, entre otros). El proceso de diseño, implementación, evaluación y ajuste de las prácticas pedagógicas se realizó a través de una prueba piloto, se desarrolló en los cursos diferentes a la población objeto de estudio y posteriormente se sometió a discusión. Luego, se implementaron primero las prácticas pedagógicas constructivas y después las practicas lúdicas, al inicio la docente leía los objetivos y explicaba la metodología de las prácticas con el fin de que los estudiantes aprendieran a desarrollarlas ellos mismos. Se conformaron grupos de cinco estudiantes que desarrollaban la estructura de las prácticas pedagógicas según la circunstancia (en forma individual y grupal). Al finalizar las prácticas pedagógicas se organizó una mesa redonda para socializar lo aprendido, vinculándolo con situaciones parecidas en el contexto local, nacional e internacional. Estas prácticas pedagógicas fueron desarrolladas con el objetivo de fortalecer las competencias ciudadanas, dado que contenían preguntas de las habilidades emocionales, cognitiva, comunicativa e integradora, con base en las temáticas de la cátedra.

En la investigación participaron dos grupos de la cátedra Formación Humanística y Ciudadanía de las facultades y programas académicos que ofreció la Universidad del Magdalena II. La muestra estuvo conformada por 150 estudiantes para validar la escala y se aplicaron las prácticas pedagógicas a 69 estudiantes; $56 \%$ de la muestra fueron mujeres y $45 \%$ fueron hombres con un rango de edad entre 16 y 28 años.

El cuestionario aplicado se construyó con base en los Estándares en Competencias Ciudadanas y las pruebas Saber del Ministerio de Educación Nacional. La escala de medida se creó siguiendo las recomendaciones para la construcción de una prueba objetiva; se llevó a cabo una revisión de la literatura para identificar las variables objeto de estudio y la formulación de ítems. Todo ello contibuyó a una primera versión de la prueba, que estuvo conformada por 60 elementos.

Enseguida, con la finalidad de depurar su longitud, se llevó a cabo un estudio analítico de la escala (McMillan y Schumacher, 2005), donde se eliminaron los ítems que presentaron baja correlación y se realizó un estudio piloto para averiguar si existe alguna dificultad en la comprensión de los ítems. A partir de los citados criterios se seleccionaron 37 ítems tipo Likert 1-7 (donde 1=total desacuerdo y $7=$ totalmente de acuerdo) tratando de explicar las cuatro habilidades que conforman las competencias ciudadanas: comunicativas (ambientes democráticos), emocionales (empatía y emociones), cognitivas (pensamiento crítico) e integradoras (acciones) (ver tabla I). Esta escala se aplicó antes y después de implementar las prácticas pedagógicas lúdicas y constructivas. 
Tabla I. Escala de Competencias ciudadanas

\begin{tabular}{|c|c|}
\hline \multicolumn{2}{|r|}{ Acciones } \\
\hline A1 & La verdadera comunicación se produce en escuchar los argumentos de los otros, sin imponer los propios. \\
\hline A2 & Hay que realizar solo los trabajos aunque tenga la posibilidad de trabajar con otro compañero. \\
\hline A3 & Prefiero dejar que otros realicen los trabajos por mí. \\
\hline A4 & Ignorar a un compañero que me pide conformar grupo de estudio favorece mi aprendizaje. \\
\hline A1 & Valoro el cumplimiento a tiempo de mis obligaciones universitarias. \\
\hline A2 & Hay que fomentar acciones que acaben con el plagio en las universidades. \\
\hline A3 & Me gusta que los compañeros que conforman grupos de estudios conmigo piensen igual que yo. \\
\hline A4 & $\begin{array}{l}\text { Creo que copiarme de las respuestas del examen de un compañero, del cuaderno o del teléfono celular, } \\
\text { enriquece mi aprendizaje. }\end{array}$ \\
\hline A5 & Me gusta participar en clase para dar a conocer mis puntos de vista sobre el tema que se está desarrollando. \\
\hline A6 & Si observo que no me escuchan mis opiniones, insisto para que mis compañeros y profesores la conozcan. \\
\hline \multicolumn{2}{|r|}{ (1) } \\
\hline E11 & No me importa perder el control cuando tengo mucha rabia. \\
\hline E12 & Me parece importante controlar la rabia y las emociones para no lastimar a las otras personas. \\
\hline E13 & Manifiesto rabia cuando agreden a un compañero y éste no hace nada para desquitarse. \\
\hline E14 & Mis acciones pueden afectar a las personas que están a mi alrededor. \\
\hline E15 & $\begin{array}{l}\text { Me gustaría que en las familias se acabaran los castigos injustos y se trabajara por la solidaridad entre sus } \\
\text { miembros. }\end{array}$ \\
\hline E16 & Me parece importante asumir una posición crítica frente a situaciones de discriminación sexual. \\
\hline E17 & $\begin{array}{l}\text { Es importante identificar las emociones que me dificultan sentir empatía por algún compañero que compiten } \\
\text { conmigo por una beca. }\end{array}$ \\
\hline E18 & El diálogo es una estrategia de conciliación que permite establecer acuerdo entre las personas implicadas. \\
\hline E19 & Las ofensas son una falta de respeto a la dignidad de las otras personas. \\
\hline E20 & Las personas deben controlar sus impulsos cuando se presentan situaciones injustas en su quehacer cotidiano. \\
\hline \multicolumn{2}{|r|}{ 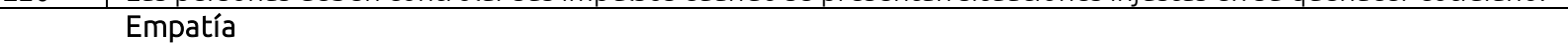 } \\
\hline Em11 & Me es fácil darme cuenta de lo que sienten las otras personas (rabia, alegría, temor) que están a mi alrededor. \\
\hline Em12 & Cuando un compañero es rechazado me siento mal; lo ayudo incluyéndolo en mi grupo de estudio. \\
\hline Em13 & Cuando un compañero sufre problemas, realizo acciones para ayudarlo. \\
\hline Em14 & Cuando mis compañeros me cuentan sus problemas me muestro indiferente. \\
\hline Em15 & Cuando se producen conflictos entre mis padres busco la manera de solucionarlo. \\
\hline Em16 & Cuando veo en televisión casos de vulneración de derechos humanos trato de ponerme en el lugar del afectado. \\
\hline Em17 & Cuando mi vecina me cuenta sus problemas familiares le ayudo planteándole varias alternativas de solución. \\
\hline Em18 & $\begin{array}{l}\text { Cuando me comunico prefiero hacerlo con gestos para expresar mis sentimientos de respeto y amor hacia las } \\
\text { otras personas. }\end{array}$ \\
\hline Em19 & Me siento mejor cuando ayudo a las personas que cuando las ignoro. \\
\hline Em20 & Mis relaciones interpersonales están fundamentadas en el respeto. \\
\hline \multicolumn{2}{|r|}{ Ambiente } \\
\hline Am11 & Es importante que los docentes escuchen las opiniones de los estudiantes. \\
\hline Am12 & e el docente estimula las relaciones democráticas en el aula. \\
\hline Am13 & Es importante que el docente proporcione un ambiente adecuado para alcanzar los objetivos propuestos. \\
\hline Am14 & Es importante que el docente considere las opiniones de sus estudiantes. \\
\hline Am15 & $\begin{array}{l}\text { Valoro la importancia de las normas que hacen posible la preservación de las diferencias culturales y regulan } \\
\text { nuestra convivencia. }\end{array}$ \\
\hline Am16 & El diálogo constructivo y un clima de confianza entre alumnos y docentes favorece el aprendizaje. \\
\hline Am17 & Los estudiantes universitarios aprenden de manera activa, posibilitando conocer sus intereses. \\
\hline Am18 & La construcción de las normas entre todos favorece la sana convivencia. \\
\hline Am19 & El ambiente de la clase favorece la adquisición de hábitos de convivencia en grupo y de respeto a los otros. \\
\hline Am20 & El docente debe ser coherente con lo que dice y hace. \\
\hline \multicolumn{2}{|r|}{ Pensamiento crítico } \\
\hline Cg6 & $\begin{array}{l}\text { Cuando no realizas los trabajos que te asignan los profesores, te da igual entregarlos a tiempo que dar una } \\
\text { excusa para poderlos entregar en una próxima oportunidad. }\end{array}$ \\
\hline $\mathrm{Cg} 7$ & $\begin{array}{l}\text { Cuando un profesor explica en clases, aunque pueda participar me muestro indiferente porque vas bien en la } \\
\text { asignatura. }\end{array}$ \\
\hline $\mathrm{Cg} 8$ & $\begin{array}{l}\text { Cuando un docente coloca tema de consulta para la próxima clase, me intereso por realizarlo, aunque no sea } \\
\text { calificable. }\end{array}$ \\
\hline Cg9 & $\begin{array}{l}\text { Cuando el docente entrega los trabajos calificados y tú no lo desarrollaste lo soluciono mintiendo, lo } \\
\text { importante es ganar la asignatura. }\end{array}$ \\
\hline Cg10 & $\begin{array}{l}\text { Cuando un compañero no realiza su trabajo autónomo le explico sin tener en cuenta los motivos de su } \\
\text { irresponsabilidad. }\end{array}$ \\
\hline
\end{tabular}




\section{Análisis y discusión de los resultados}

\subsection{Validación de la escala}

Se realizó un Análisis Factorial Confirmatorio a la escala propuesta, quitando aquellos que no influían en la construcción de la variable latente ( $p>0.10)$. La nueva escala constó de 29 ítems tipo Likert que incluye las habilidades integradoras (3 ítems acciones), comunicativa (9 ítems ambientes democráticos), emocionales ( 7 ítems emociones y empatía) y cognitiva (3 ítems pensamiento crítico), con un alfa de Cronbach de 0.73 para la escala total. Los datos de la escala se registran en la tabla II. 
Tabla II. Propiedades psicométricas de las escalas

\begin{tabular}{|c|c|c|c|c|}
\hline Variable & ítems & $\begin{array}{c}\text { B } \\
\text { (t valor) }\end{array}$ & E-esta & $\mathrm{P}$ \\
\hline \multirow{3}{*}{$\begin{array}{l}\text { Acciones } \\
\text { (ICFES, 2005) }\end{array}$} & $\begin{array}{l}\text { A1: La verdadera comunicación es aquella que se produce en escuchar los argumentos de los otros, sin imponer } \\
\text { los propios. }\end{array}$ & $0.44(1.76)$ & 0.25 & 0.07 \\
\hline & A2: Me gusta participar en clase para dar a conocer mis puntos de vista sobre el tema que se está desarrollando. & $1.74(11.66)$ & 0.15 & 0.00 \\
\hline & A3: Si observo que no me escuchan mis opiniones, insisto para que mis compañeros y profesores la conozcan. & $1.07(4.99)$ & 0.21 & 0.00 \\
\hline \multirow{7}{*}{$\begin{array}{l}\text { Emociones } \\
\text { (ICFES, 2005) }\end{array}$} & E1: No me importa perder el control cuando tengo mucha rabia. & $0.55(3.88)$ & 0.14 & 0.00 \\
\hline & E2: Mis acciones pueden afectar a las personas que están a mi alrededor. & $0.88(5.64)$ & 0.15 & 0.00 \\
\hline & E3: Me parece importante asumir una posición crítica frente a situaciones de discriminación sexual. & $0.50(3.42)$ & 0.15 & 0.00 \\
\hline & $\begin{array}{l}\text { E4: Es importante identificar las emociones que me dificultan sentir empatía por algún compañero que compite } \\
\text { conmigo por una beca. }\end{array}$ & $0.92(5.11)$ & 0.18 & 0.00 \\
\hline & E5: El diálogo es una estrategia de conciliación que permite establecer acuerdo entre las personas implicadas. & $0.65(3.70)$ & 0.17 & 0.00 \\
\hline & E6: Las ofensas son una falta de respeto a la dignidad de las otras personas. & $0.84(5.66)$ & 0.14 & 0.00 \\
\hline & $\begin{array}{l}\text { E7: Las personas deben controlar sus impulsos cuando se les presentan situaciones injustas en su quehacer } \\
\text { cotidiano. }\end{array}$ & $0.94(6.01)$ & 0.15 & 0.00 \\
\hline \multirow{7}{*}{$\begin{array}{l}\text { Empatía } \\
\text { (ICFES, 2004- } \\
2005)\end{array}$} & $\begin{array}{l}\text { Em1: Me es fácil darme cuenta de lo que sienten las otras personas (rabia, alegría, tristeza temor) que están a mi } \\
\text { alrededor. }\end{array}$ & $0.56(3.80)$ & 0.14 & 0.00 \\
\hline & Em2: Cuando un compañero es rechazado me siento mal; lo ayudo incluyéndolo en mi grupo de estudio. & $0.64(4.39)$ & 0.14 & 0.00 \\
\hline & Em3: Cuando un compañero sufre problemas realizo acciones para ayudarlo. & $0.69(4.29)$ & 0.16 & 0.00 \\
\hline & Em4: Cuando mis compañeros me cuentan sus problemas me muestro indiferente. & $0.66(3.17)$ & 0.21 & 0.00 \\
\hline & $\begin{array}{l}\text { Em5: Cuando veo en la televisión casos de vulneración de derechos humanos trato de ponerme en el lugar del } \\
\text { afectado. }\end{array}$ & $0.82(4.92)$ & 0.16 & 0.00 \\
\hline & $\begin{array}{l}\text { Em6: Cuando me comunico prefiero hacerlo con gestos para expresar mis sentimientos de respeto y amor hacia } \\
\text { las otras personas. }\end{array}$ & $0.97(4.99)$ & 0.19 & 0.00 \\
\hline & Em7: Me siento mejor cuando ayudo a las personas que cuando las ignoro. & $0.39(3.43)$ & 0.11 & 0.00 \\
\hline \multirow{9}{*}{$\begin{array}{l}\text { Ambiente } \\
\text { (MEN, 2004) }\end{array}$} & Am1: Es importante que el docente estimula las relaciones democráticas en el aula. & $0.58(3.09)$ & 0.18 & 0.00 \\
\hline & Am2: Es importante que el docente proporcione un ambiente adecuado para alcanzar los objetivos propuestos. & $0.71(3.64)$ & 0.19 & 0.00 \\
\hline & Am3: Es importante que el docente considere las opiniones de sus estudiantes. & $0.46(2.73)$ & 0.16 & 0.01 \\
\hline & $\begin{array}{l}\text { Am4: Valoro la importancia de las normas que hacen posible la preservación de las diferencias culturales y } \\
\text { regulan nuestra convivencia. }\end{array}$ & $0.42(2.25)$ & 0.187 & 0.02 \\
\hline & Am5: El diálogo constructivo y un clima de confianza entre alumnos y docentes favorece el aprendizaje. & $0.58(4.32)$ & 0.13 & 0.00 \\
\hline & Am6: Los estudiantes universitarios aprenden de manera activa, posibilitando conocer sus intereses. & $0.96(6.58)$ & 0.14 & 0.00 \\
\hline & Am7: La construcción de las normas entre todos favorece la sana convivencia. & $0.48(2.66)$ & 0.18 & 0.01 \\
\hline & Am8: El ambiente de la clase favorece la adquisición de hábitos de convivencia en grupo y de respeto a los otros. & $0.99(6.18)$ & 0.16 & 0.00 \\
\hline & Am9: El docente debe ser coherente con lo que dice y hace. & $0.57(4.03)$ & 0.14 & 0.00 \\
\hline \multirow{3}{*}{$\begin{array}{l}\text { Pensamiento } \\
\text { Crítico } \\
\text { (Ruiz y } \\
\text { Chaux, 2005, } \\
\text { Soriano, } \\
2006 \text { ) } \\
\end{array}$} & $\begin{array}{l}\text { Cg1: Cuando un docente coloca tema de consulta para la próxima clase, me intereso por realizarlo, aunque no } \\
\text { sea calificable. }\end{array}$ & $0.39(2.63)$ & 0.14 & 0.01 \\
\hline & $\begin{array}{l}\text { Cg2: Cuando el docente entrega los trabajos calificados y tú no lo desarrollaste, lo soluciono mintiendo, lo } \\
\text { importante es ganar la asignatura. }\end{array}$ & $0.83(3.11)$ & 0.26 & 0.00 \\
\hline & $\begin{array}{l}\text { Cg3: Cuando un compañero no realiza su trabajo autónomo, le explico sin tener en cuenta los motivos de su } \\
\text { irresponsabilidad. }\end{array}$ & $0.62(2.69)$ & 0.23 & 0.07 \\
\hline
\end{tabular}


Los resultados se presentan a través de un análisis inferencial, a través de la Prueba Anova, utilizando el modelo lineal general, en el que la variable dependiente fueron los diferentes elementos de las competencias ciudadanas, y la variable independiente las prácticas pedagógicas constructivas (estudios de casos) y lúdicas (juegos), frente a las prácticas expositivas (clases magistrales).

Con este procedimiento se contrastó la $\mathrm{H} 1$, que proponía que las acciones ciudadanas son significativamente mayores en el grupo experimental que ha recibido prácticas pedagógicas constructivas y lúdicas, que en el grupo de control, que ha recibido prácticas pedagógicas expositivas. Los resultados muestran que los tipos de práctica pedagógica influyen en el resultado que se obtiene en las acciones ciudadanas ( $\mathrm{p} \leq 0.05)$ (ver tabla III).

Tabla III. Efecto de las prácticas pedagógicas lúdicas y constructivas sobre las competencias ciudadanas

\begin{tabular}{l|c|c|c|c}
\hline Competencias ciudadanas & G1 & G2 & F & P \\
\hline Acciones ciudadanas & 14.64 & 16.67 & 8.27 & 0.00 \\
Ambiente & 40.87 & 49.17 & 26.91 & 0.00 \\
Emociones & 36.66 & 40.75 & 6.87 & 0.01 \\
Empatía & 37.00 & 38.34 & 0.87 & 0.35 \\
Congnitiva & 19.00 & 20.87 & 7.42 & 0.00 \\
\hline
\end{tabular}

Normalidad: No se observan importantes desviaciones respecto a la normalidad.

G1: Grupo que recibió prácticas pedagógicas expositivas.

G2: Grupo que recibió prácticas pedagógicas constructivas y lúdicas

A la luz de estos resultados se puede afirmar que el desarrollo de las acciones ciudadanas está influenciado por el tipo de prácticas pedagógicas que se utilicen y se confirma la hipótesis H1 (figura 1).

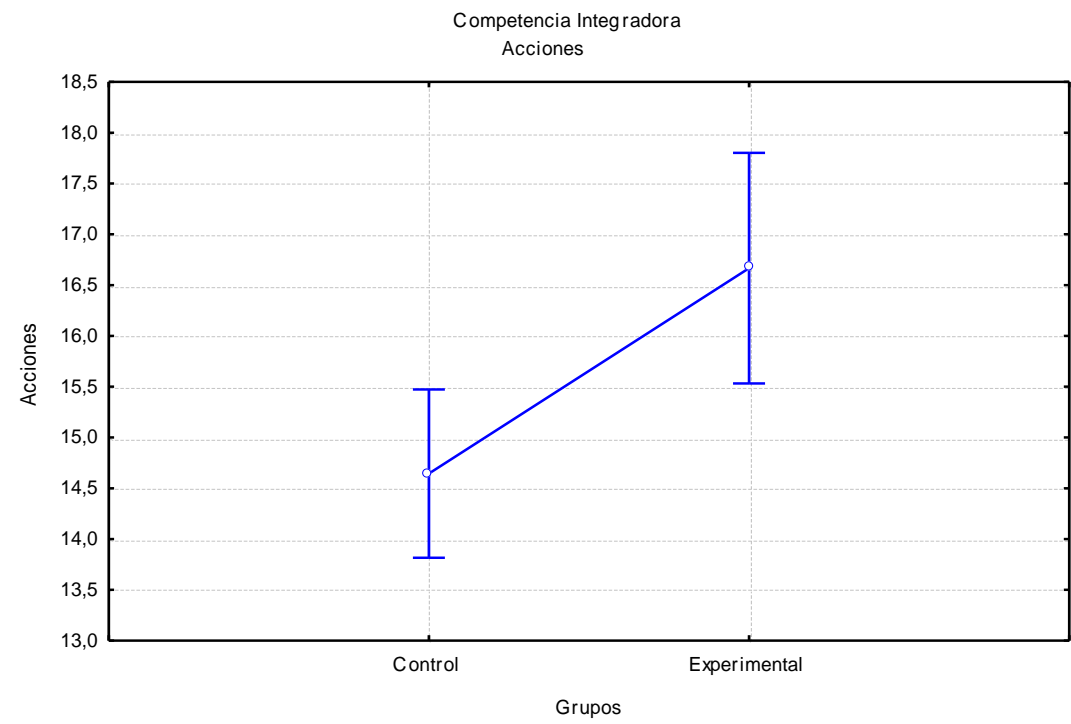

Figura 1. Medias de acciones entre el grupo de control y el experimental

Los resultados corroboran las investigaciones de Kohlberg (1984), quien señala que la acción que el sujeto realiza está determinada por los valores que han adquirido en el entorno social y que con base en ellos se actúa en la experiencia diaria, desarrollando cada vez interacciones de mayor complejidad, producto del aprendizaje lógico-evolutivo que generan los procesos de la acción comunicativa (Habermas, 1981). Esto demuestra que las acciones ciudadanas están relacionadas con la motivación, la cual encaminan las emociones hacia acciones conscientes, dando lugar a "esfuerzo intelectual y físico, con el fin de lograr unas metas previamente establecidas" (Williams y Burden 1999, p. 128). 
Continuando con $\mathrm{H} 2$, que proponía que las prácticas pedagógicas influían en los ambientes democráticos, siendo mayores en aquellos alumnos que habían recibido prácticas pedagógicas constructivas y lúdicas, frente a los que habían recibido prácticas pedagógicas expositivas, podemos decir que los resultados del Anova muestran un efecto principal significativo para la competencia comunicativa en ambientes democráticos ( $p \leq 0.05)$, siendo dichos resultados mayores en el grupo experimental (49.17) que en el grupo de control (40.87) (ver tabla III). Estos hallazgos permiten confirmar definitivamente la $\mathrm{H} 2$, concluyendo que la práctica pedagógica utilizada es fundamental para el desarrollo de un ambiente democrático (ver figura 2).

Estos hallazgos son coherentes con las investigaciones de Robles (2011), quien señala que los individuos pasan por diferentes instituciones y ámbitos que les facilitan la transmisión de los conocimientos y valores, desarrollar competencias y capacidades que les permiten vivir como sujetos autónomos. En este caso la explicación está en el ambiente de aprendizaje en que se desarrollaron los juegos y estudios de casos, ya que estos son activos, acogedores y formadores, generando en los estudiantes opciones para hacer que sus acciones sean más coherentes con sus valores, su sentir y pensar, con lo que se busca el crecimiento de las dimensiones sociales, cognitivas y emocionales de su ser (Sacristán, 2001), coincidiendo con lo planteado por Prodócimo, Goncalves, Rodríguez y Bognolí (2014), quienes consideran que el ambiente del aula contribuye al bienestar necesario para un buen aprendizaje y la tranquilidad emocional.

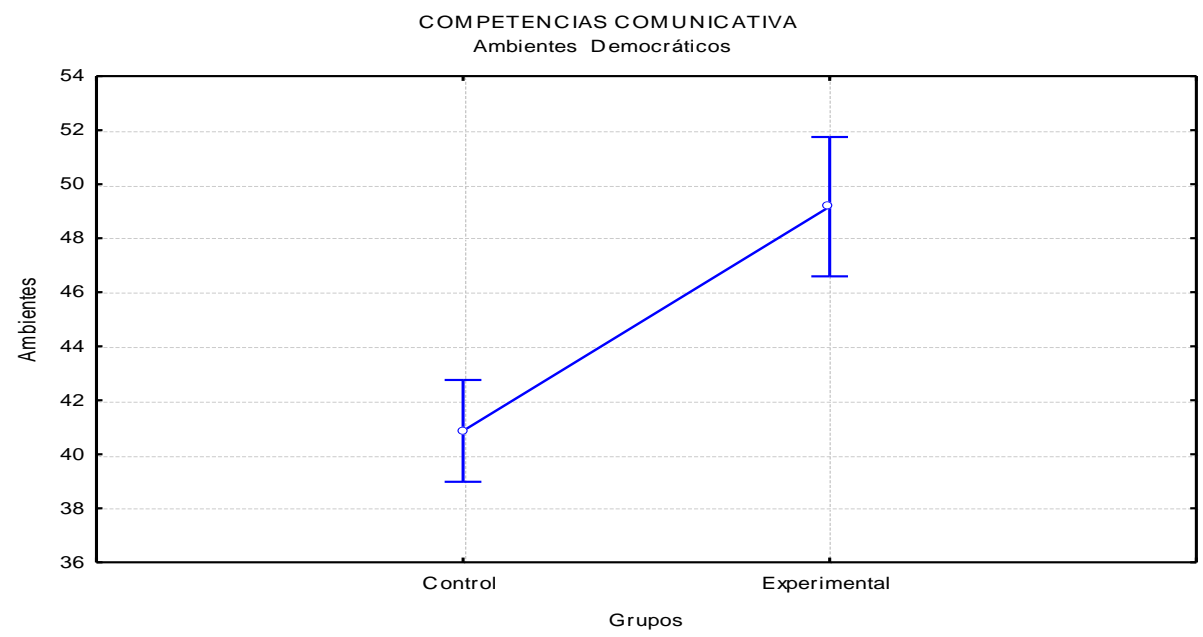

Figura 2. Medias de ambiente entre el grupo de control y el experimental

La H3 propone que las emociones en el grupo experimental que ha recibido prácticas pedagógicas constructivas y lúdicas son significativamente mayores que en el grupo de control, que ha recibido prácticas pedagógicas expositivas. Esto significa que los estudiantes han adquirido habilidades y actitudes para regular sus emociones (rabia, alegría, tristeza, entre otras), vivenciar los sentimientos y analizar sus propias emociones y las de los demás. Los resultados de Anova muestran que existe una diferencia significativa entre los grupos en los resultados obtenidos en emociones $(p \leq 0.05)$, siendo dicho resultado mayor en el grupo experimental (40.75) que en el grupo de control (36.66) (ver tabla Ill y figura 3). Estos hallazgos permiten confirmar definitivamente la $\mathrm{H} 3$, ratificando que las prácticas pedagógicas lúdicas y constructivas permiten que los estudiantes reconocieron sus emociones e identificaron el tipo de emoción que han experimentado ellos mismos y sus compañeros; consolidándose en los estudiantes inteligencia intrapersonal e interpersonal (Gardner,1993). 


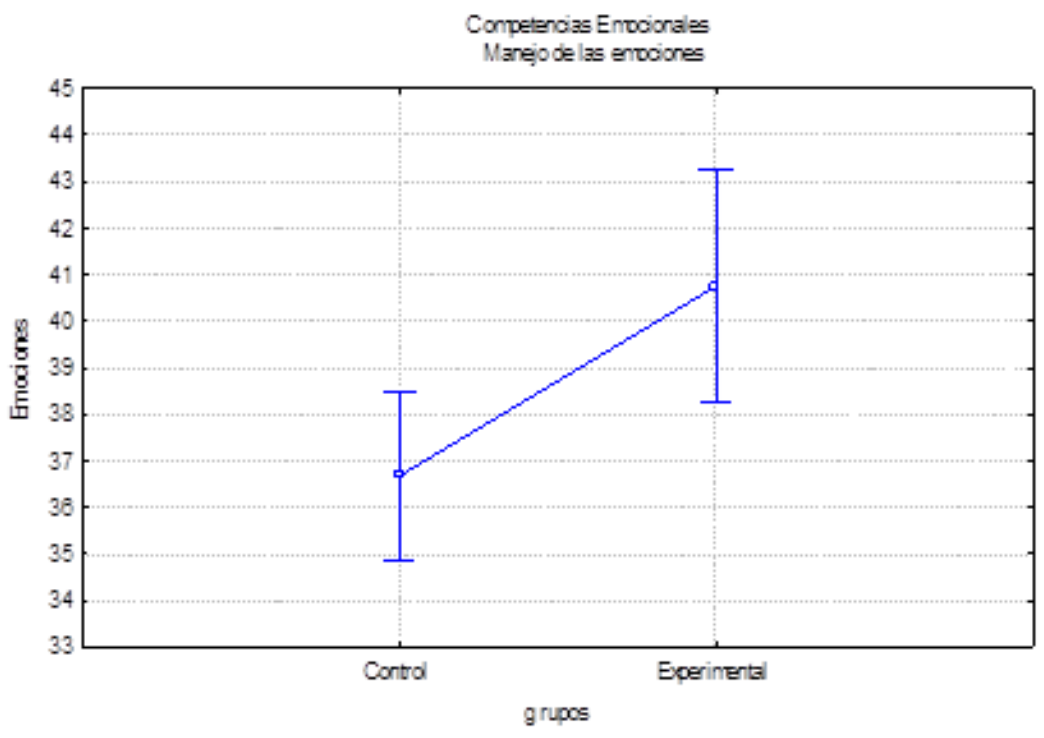

Figura 3. Medias de emociones entre el grupo control y el experimental

La H4 se centraba en la empatía y proponía resultados diferentes en esta variable entre los diferentes grupos; mayor en el grupo experimental que para el grupo de control. En este caso, la Anova muestra diferencias no significativas ( $p \geq 0.10$ ), siendo dichos resultados muy similares entre el grupo experimental (38.34) con los del grupo de control (37.00) (ver tabla III y figura 4). Estos hallazgos son contrarios a lo establecido en la H4, por lo que la hipótesis no se puede confirmar.

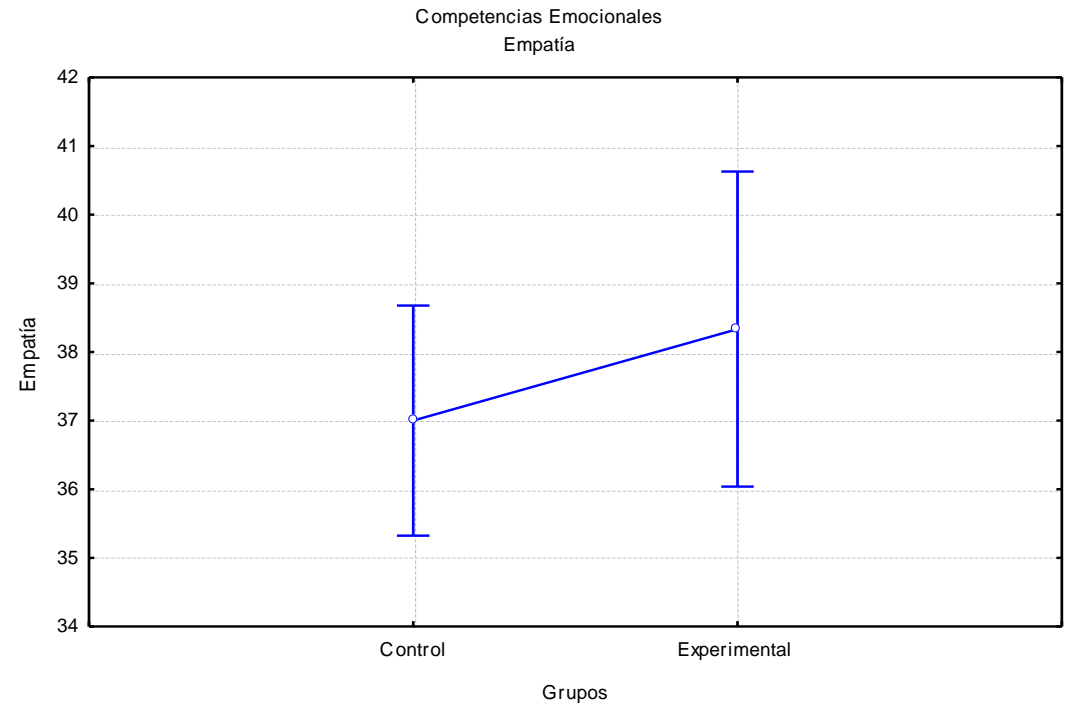

Figura 4. Medias de empatía entre el grupo control y el experimental

Una posible causa se debe a lo expresado por Singer y Lamm (2009) que consideraban que esta habilidad es flexible y depende de una gran cantidad de factores como el contexto, de la relación interpersonal con el otro y la perspectiva que se adopta al observar las circunstancias y los intereses de la persona a quien ayuda (Clark, 1980) y la educación que han recibido los jóvenes por parte de sus padres (Batson, 1991). Por lo tanto, sus respuestas afectivas van a variar según las situaciones y las personas con quienes interactúan. 
Por último, la H5 proponía que el pensamiento crítico es significativamente mayor en el grupo experimental que ha recibido prácticas pedagógicas constructivas y lúdicas que en el grupo de control, que ha utilizado prácticas pedagógicas expositivas. De nuevo, los resultados del Anova muestran una diferencia significativa en el pensamiento crítico $(p \leq 0.05)$ entre los grupos, siendo dicho resultado mayor en el experimental (20.87) que en el de control (19.00) (ver tabla Ill y figura 5). Estos hallazgos permiten confirmar la H5.

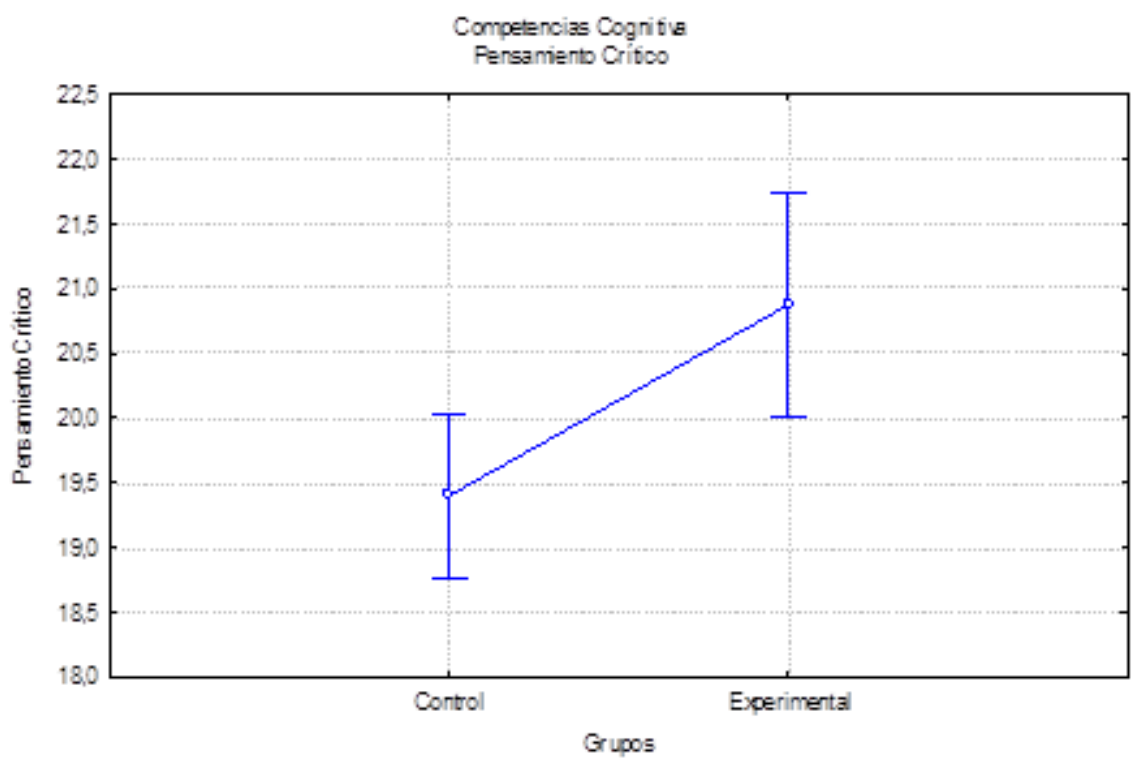

Figura 5. Medias de pensamiento crítico entre el grupo control y el experimental

Estos hallazgos son coherentes con las investigaciones de Villarini (1987), quien considera que el pensamiento crítico surge de la metacognición, desarrollando las capacidades de dimensión lógica, dimensión sustantiva, dimensión contextual, dimensión dialógica y dimensión pragmática, aspectos esenciales en el ámbito del pensamiento crítico a nivel universitario.

\section{Conclusiones}

En este trabajo se examinó la influencia de las prácticas pedagógicas (estudios de casos y juegos) utilizadas en el aula sobre el desarrollo de las competencias ciudadanas en un grupo de estudiantes universitarios, concluyendo que la aplicación de estas prácticas pedagógicas son más eficaces que las prácticas pedagógicas expositivas (clases magistrales). Lo que explica los resultados obtenidos son la naturaleza del programa de intervención de las prácticas pedagógicas (constructivas), la etapa en la que se encontraban los sujetos objeto de investigación (Arón y Milicic, 1999) y la relación de esta competencia con la habilidad cognitiva (Lane y Schwart, 1987).

Con respecto a los resultados obtenidos en la empatía, es importante señalar la necesidad evaluar su impacto a través de opciones metodológicas integradas desde el ejercicio de la ciudadanía (CalderíusFernández y Martínez-Sánchez, 2013); es decir, desde la actuación. Esto se debe a que es una práctica y una vivencia (Zuta, Velasco y Rodríguez, 2014). El uso de diferentes estrategias y procedimientos, como la observación, la entrevista y el portafolio, nos permite recabar y analizar la información, evaluando los avances de los estudiantes y haciendo los ajustes para alcanzar el propósito establecido. La aplicación de la evaluación integral de los aprendizajes requerirá de esfuerzo prolongado y consistente de los maestros para mejorar sus prácticas de evaluación en el aula (Martínez-Rizo y Mercado, 2015). 
El análisis de los datos en el componente ambiente, emociones y pensamiento crítico ha puesto en evidencia que las prácticas pedagógicas expositivas (clases magistrales) no permiten el desarrollo de las competencias ciudadanas, ya que el estudiante no puede manifestar sus emociones, reflexionar sobre sus experiencias vividas, expresar sus puntos de vistas, desarrollar la habilidad comunicativa de la asertividad ni poner en prácticas los conocimientos, valores y acciones ciudadanas en el aula. Lo anterior coincide con lo planteado por Ruiz y Chaux (2005, p. 49): "El contexto puede obstaculizar o favorecer el ejercicio de esta competencia ciudadana".

Una limitación de esta investigación es el tiempo reducido para la aplicación de las prácticas lúdicas (juegos) y las prácticas constructivas (estudio de casos), debido a la obligación de cumplir el microcurrículo que regula los contenidos de la cátedra "Formación Humanística y Ciudadana". Si hubiéramos contado con mayor tiempo para implementar estas prácticas pedagógicas la experiencia hubiera tenido más éxito y los estudiantes podrían haber elaborado ellos mismos prácticas lúdicas (juegos didácticos) y prácticas constructivas (estudios de casos) para todos los contenidos desarrollados.

Por último, aunque reconocemos la utilidad y pertinencia de este estudio, es necesario que futuras investigaciones indaguen cuál de las prácticas pedagógicas (lúdicas o constructivas) son más pertinentes en el contexto universitario y contribuyen en el desarrollo de las competencias ciudadanas. Además, estas intervenciones se podrían realizar de manera simultánea en varios de los sistemas (meso, micro, macro y exosistema) (Bronfenbrenner, 1987), lo que conllevaría abrir espacios en las instituciones universitaria para que los estudiantes desarrollen autonomía e independencia de pensamiento y de acción.

\section{Referencias}

Arón, A. y Milicic, N (1999). Vivir con otros. Programa de desarrollo de habilidades sociales. Santiago de Chile: Ediciones Universitaria.

Batson, D. (1991). The altruism question: toward a social-psychological answer. Nueva Jersey: Lawrence Erlbaum Associates.

Baumeister, R. y Heatherton,T. (1996). Sef-regulation failure: an overview. Psychological Inquiry, 7(1),1-15.

Bisquerra, R. (2000). Educación emocional y bienestar. Barcelona: Praxis.

Briones, G. (2004). La investigación en el aula y en la escuela. Formación de docentes en investigación educativa (Módulo 2). Colombia: Convenio Andrés Bello.

Bronfenbrenner, U. (1987). La ecología del desarrollo humano. Barcelona: Paidós.

Cano, B. (2004). Pedagogía y didáctica de la educación ética y los valores humanos. Colombia: Paulina.

Calderíus-Fernández, M. y Martínez-Sánchez, N. (2013). La Formación de competencias ciudadanas en las universidades cubanas. Revista Santiago, 132, 687-713.

Carrillo, O. (2013). Comprendiendo la adquisición de las competencias ciudadanas en alumnos de los programas de cualificación profesional inicial. Revista Educar, 49(2), 207-226.

Climent, J. (2010). Reflexiones sobre la educación basada en competencias. Revista Complutense de Educación, 21(1), 91-106.

Clark, K. B. (1980). Empathy: a neglected topic in psychological research. American Psychologist, 35(2),187190.

Gardner, H.(1983). Estructura de la mente: teoría de las inteligencias múltiples. Madrid: Fondo de Cultura Económica. 
Gardner, H. (1993). Inteligencias múltiples de la teoría a la práctica. Barcelona: Paidós.

Giroux, H. (1992). La pedagogía de frontera y la política del postmodernismo. Revista Intríngulis, 6, 33-47.

Gros, B. y Contreras, D. (2006). La alfabetización digital y el desarrollo de competencias ciudadanas. Revista Iberoamericana de Educación, 42, 103-125.

Habermas, J. (1981). La reconstrucción del materialismo histórico. Madrid:Taurus.

Kohlberg, L. (1984). The psychology of moral development: the nature and validity of moral stages. EUA: Harper \& Row.

Instituto Colombiano para el Fomento de la Educación Superior. (2004). Prueba Saber en competencias ciudadanas. Bogotá, Colombia: Autor.

Instituto Colombiano para el Fomento de la Educación Superior. (2005). Guía de orientación de competencias. Bogotá, Colombia: Autor.

Lane, R. D. y Schwartz, G. E. (1987). Levels of emotional awareness: a cognitive-developmental theory and its application to psychopathology. American Journal of Psychiatry, 144(2), 133-144.

Martínez-Rizo, F. y Mercado, A. (2015). Estudio sobre prácticas de evaluación en el aula: revisión de la literatura. Revista Electrónica de Investigación Educativa, 17(1),17-32. Recuperado de

https://redie.uabc.mx/redie/article/view/371

McMillan, J. y Schumacher, S. (2005). Investigación educativa. Una introducción conceptual (5a. ed). Madrid: Pearson.

Mieles, M. y Alvarado, S. (2012). Ciudadanía y Competencias ciudadana. Estudios Políticos, 40, 53-75. Recuperado de

http://aprendeenlinea.udea.edu.co/revistas/index.php/estudiospoliticos/article/view/13203/20779249

Ministerio de Educación de Colombia. (2004). Formar para la ciudadanía ¡Sí es posible! Bogotá, Colombia: Autor.

Ministerio de Educación de Colombia. (2005). Taller internacional de formación en competencias ciudadanas. Bogotá, Colombia: Autor.

Ministerio de Educación de Colombia. (2006). Estándares básicos de competencias en Lenguaje, matemática, ciencias y ciudadanía. Bogotá: Autor.

Puig, J. (1996). La construcción de la personalidad moral. Barcelona: Paidós.

Prodócimo, E., Goncalves, R., Rodríguez R. y Bognolí P. (2014), Violencia escolar: reflexiones sobre los espacios de ocurrencia. Revista Electrónica de Investigación Educativa,16(2),1-15. Recuperado de https://redie.uabc.mx/redie/article/view/513

Rangel, H. (2015). Una mirada internacional de la construcción curricular. Por un currículo vivo, democrático y deliberativo. Revista Electrónica de Investigación Educativa,17(1),1-16. Recuperado de https://redie.uabc.mx/redie/article/view/380

Robles, M. (2011). Investigaciones internacionales sobre la implicación cívica de adolescentes y jóvenes. Ciudadanos adolescentes en la era digital. Revista Electrónica Interuniversitaria de Formación del Profesorado, 14 (2) 71-79. 
Ruiz, A. y Chaux, E. (2005). La formación en competencias ciudadanas. Colombia: Ascofade.

Sacristán, J. (2001). Educar y convivir en la cultura global. Madrid, España: Morata.

Singer, T. y Lamm, C.(2009). The social neuroscience of empathy [El enfoque social neurocientífico de la empatía]. Annals of the New York Accademy of Sciences, 1156, 81-96.

Soriano, E. (2006). Competencias Ciudadanas en el alumnado de segundo siclo de educación secundaria obligatoria de Almeria. Revista de Investigación Educativa, 24(1),119-146.

Tey, A., Vilá, R. y Martín, M. (2014). Competencias para el aprendizaje ético en estudiantes universitario de enfermeria y pedagógia. Revista de docencia universitaria, 12(1), 337- 352.

Ugarriza, N. y Pajares, L. (2005). La evaluación de la inteligencia emocional a través del inventario de BarOn (I-CE) en una muestra de niños y adolecentes. Persona, 8, 11-58.

Ugarriza, N. (2001). La evaluación de la inteligencia emocional a través del inventario de BarOn (I-CE) en una muestra de Lima Metropolitana. Persona, 4, 129-160.

Villarini, A. (1987). Principios para la integración del currículo. Puerto Rico: Departamento de Instrucción Pública.

Villarini, A. (2010). Competentes para la democracia. Una propuesta alternativa de formación ciudadana. Puerto Rico: Organización para el Fomento del Desarrollo del Pensamiento.

Williams, M y Burden, R.L.(1999). Psicología para profesores de Idiomas. Enfoque de Constructivismo social. Madrid, España:Cambridge University.

Zambrano, E. Rivera, A., Fernández, F. y González, R. (2014). La práctica pedagógica constructiva: el método de caso. Memorias, 12(22) 81-92.

Zuta, E.,Velasco, A. y Rodríguez, J. (2014). Desarrollo de competencias ciudadanas mediante un curso socialmente. Revista Educación, 23(45), 51-66. 